\title{
Cosmological constraints on induced gravity dark energy models
}

\author{
M. Ballardini ${ }^{a, b, c}$ F. Finelli ${ }^{b, c}$ C. Umiltà ${ }^{d, e, f}$ and D. Paoletti ${ }^{b, c}$ \\ ${ }^{a}$ DIFA, Dipartimento di Fisica e Astronomia, Alma Mater Studiorum Università di Bologna, \\ Viale Berti Pichat 6/2, I-40127 Bologna, Italy \\ ${ }^{b}$ INAF-IASF Bologna, via Gobetti 101, I-40129 Bologna, Italy \\ ${ }^{c}$ INFN, Sezione di Bologna, Via Irnerio 46, I-40126 Bologna, Italy \\ ${ }^{d}$ Institut d'Astrophysique de Paris, CNRS (UMR7095), 98 bis Boulevard Arago, F-75014, \\ Paris, France \\ ${ }^{e}$ UPMC Univ Paris 06, UMR7095, 98 bis Boulevard Arago, F-75014, Paris, France \\ ${ }^{f}$ Sorbonne Universités, Institut Lagrange de Paris (ILP), 98 bis Boulevard Arago, 75014 \\ Paris, France \\ E-mail: ballardini@iasfbo.inaf.it, finelli@iasfbo.inaf.it, umilta@iap.fr, \\ paoletti@iasfbo.inaf.it
}

\begin{abstract}
We study induced gravity dark energy models coupled with a simple monomial potential $\propto \sigma^{n}$ and a positive exponent $n$. These simple potentials lead to viable dark energy models with a weak dependence on the exponent, which characterizes the accelerated expansion of the cosmological model in the asymptotic attractor, when ordinary matter becomes negligible. We use recent cosmological data to constrain the coupling $\gamma$ to the Ricci curvature, under the assumptions that the scalar field starts at rest deep in the radiation era and that the gravitational constant in the Einstein equations is compatible with the one measured in a Cavendish-like experiment. By using Planck 2015 data only, we obtain the $95 \%$ CL bound $\gamma<0.0017$ for $n=4$, which is further tightened to $\gamma<0.00075$ by adding Baryonic Acoustic Oscillations $(\mathrm{BAO})$ data. This latter bound improves by $\sim 30 \%$ the limit obtained with the Planck 2013 data and the same compilation of BAO data. We discuss the dependence of the $\gamma$ and $\dot{G}_{N} / G_{N}(z=0)$ on $n$.
\end{abstract}




\section{Contents}

1 Introduction 1

2 Dark Energy with a monomial potential within Induced Gravity 2

3 Linear fluctuations and predictions for cosmological observables $\quad 3$

4 Predictions for CMB anisotropies and Matter Power spectrum 5

5 Updated Planck 2015 results for the quartic potential 6

5.1 Combination with local measurements 11

$\begin{array}{lll}5.2 & \mathrm{BBN} \text { consistency relation on } G_{\mathrm{N}} & 11\end{array}$

6 Cosmological constraints for $n \neq 4 \quad 12$

\begin{tabular}{llr}
7 & Conclusions & 12 \\
\hline
\end{tabular}

\section{Introduction}

The most recent data from PLANCK [1, 2] are consistent with a cosmological constant and cold dark matter $(\mathrm{CDM})$ as the dark components which contribute to the $96 \%$ of the energy budget of our Universe. Several dark energy/modified gravity models alternative to $\Lambda$ CDM model have been compared extensively to the most recent Planck data [3]. The predictions for CMB anisotropies and for other cosmological observabels for these modified gravity scenarios can be studied either in a model-dependent way or by more general parametrized deviations from Einstein gravity in the metric perturbations [4]. By using both approaches, no compelling evidence in favour of alternative models to $\Lambda \mathrm{CDM}$ have been found in [3], in particular when Planck lensing is included [5]. Other parametrized deviations from Einstein gravity in extended cosmological models have been studied in [6].

In this paper we study the simplest scalar-tensor dark energy models based on induced gravity (IG) [7] - or Brans-Dicke-like models [8] by a redefinition of the scalar field - with a monomial potential, extending previous works based on a quartic potential [9-12] (see also [13-18] for important works on scalar-tensor dark energy). By assuming a monomial potential with a positive exponent $n$ - i.e. $V(\sigma) \propto \sigma^{n}$ - mimicking an effective cosmological constant at recent times, the scalar field is frozen during the radiation era and is driven by the nonrelativistic components after the matter-radiation equality to higher values: in these models the effective Planck mass therefore increase in time. Such monomial potentials with a positive power are easily motivated at fundamental level or within particle physics, as happens for the analogous case of a non-minimally coupled scalar field [19].

We extend our self-consistent approach in which we solve simultaneously the background and the linear perturbation dynamics in IG previously applied to a quartic potential [12]. This approach is complementary to the study of parametrized deviations from Einstein gravity in metric perturbations, since it allows to accurately study modified gravity theoretical models which are arbitrary close to the flat $\Lambda \mathrm{CDM}$, without any approximation in the background or in the perturbations. We then use recent cosmological data to constrain these IG models with a monomial potential. In a previous study we obtained a $95 \%$ CL bound $\gamma<0.0012$ 
for a quartic potential by the Planck nominal mission temperature data in combination with a BAO compilation [12].

Our paper is organized as follows. In the next section we discuss the background dynamics and the dependence of the self-accelerating solutions on the exponent of the monomial potential. In section 3 we discuss the evolution of linear fluctuations and we test our numerical treatment against the analytic approximations in the quasi-static regime, as done for the quartic potential in [12]. We show the dependence of the CMB anisotropies power spectra in temperature and polarization on the power of the monomial potential in section 4 . We then use the Planck $2015[5,20]$ and BAO [21-23] data to constrain these models in section 5 and 6. We draw our conclusions in section 7 .

\section{Dark Energy with a monomial potential within Induced Gravity}

The model we consider is described by the following Lagrangian:

$$
S=\int d^{4} x \sqrt{-g}\left[\frac{\gamma \sigma^{2} R}{2}-\frac{g^{\mu \nu}}{2} \partial_{\mu} \sigma \partial_{\nu} \sigma-V(\sigma)+\mathcal{L}_{m}\right]
$$

with

$$
V(\sigma)=\lambda_{n} \sigma^{n} .
$$

The Friedmann and the Klein-Gordon equations for IG in a flat Robertson-Walker metric are respectively:

$$
\begin{gathered}
H^{2}+2 H \frac{\dot{\sigma}}{\sigma}=\frac{\sum_{i} \rho_{i}+V(\sigma)}{3 \gamma \sigma^{2}}+\frac{\dot{\sigma}^{2}}{6 \gamma \sigma^{2}} \\
\ddot{\sigma}+3 H \dot{\sigma}+\frac{\dot{\sigma}^{2}}{\sigma}+\frac{1}{(1+6 \gamma)}\left(V_{, \sigma}-\frac{4 V}{\sigma}\right)=\frac{1}{(1+6 \gamma)} \frac{\sum_{i}\left(\rho_{i}-3 p_{i}\right)}{\sigma}
\end{gathered}
$$

once the Einstein trace equation:

$$
-\gamma \sigma^{2} R=T-(1+6 \gamma) \partial_{\mu} \sigma \partial^{\mu} \sigma-4 V-6 \gamma \sigma \square \sigma
$$

is used. In the above $V_{, \sigma}$ denotes the derivative of the potential $V(\sigma)$ with respect to $\sigma$, the index $i$ runs over all fluid components, i.e. baryons, cold dark matter (CDM), photons and neutrinos, and we use a dot for the derivative with respect to the cosmic time. The effective potential in Eq. 2.4 vanishes for $n=4$.

In absence of matter, exact solutions with an accelerated expansion exist for this class of monomial potentials in Eq. 2.2 within induced gravity [24] (for earlier works see Ref. [25]). Solutions with $a(t) \sim t^{p}$ (with $t>0$ and $p>1$ ) exist:

$$
p=2 \frac{1+(n+2) \gamma}{(n-4)(n-2) \gamma}, \quad \sigma(t)=\frac{c_{0}}{t^{\frac{2}{(n-2)}}},
$$

with $4<n<4+\sqrt{2(6+1 / \gamma)}$ or $4-\sqrt{2(6+1 / \gamma)}<n<2$ and $c_{0}$ is an integration constant. The special cases with $n=2,4$ (which correspond to poles in the above equations) correspond to a de Sitter solution having $a(t) \propto e^{H t}$. However for these two special cases, the time evolution for the scalar field are different, being $\sigma$ time dependent for $n=2$ and constant in time for $n=4$.

As in [12], we consider the case in which the scalar field $\sigma$ at rest deep in the radiation era, since an initial non-vanishing time derivative would be rapidly dissipated [11]. For values 
for the parameters of the potential leading to viable models of dark energy, the scalar is effectively massless during the radiation and most of the matter era. The scalar field $\sigma$ starts evolving form its initial value $\sigma_{i}$ due the presence of non-relativistic matter:

$$
\sigma(\tau)=\sigma_{i}\left(1+\frac{3}{2} \gamma \omega \tau+\mathcal{O}\left(\tau^{2}\right)\right)
$$

where the parameter $\omega$ depends on the relativistic and non-relativistic energy density at present:

$$
\omega=\frac{\rho_{m 0}}{\sqrt{3 \gamma \rho_{r 0} 0}(1+6 \gamma) \sigma_{i}} .
$$

At the same next-to-leading order in $\omega \tau$ for a Universe filled by radiation and matter, the scale factor is:

$$
a(\tau)=\frac{\rho_{r 0}}{\sqrt{3 \gamma} \sigma_{i}} \tau\left(1+\frac{\omega}{4} \tau-\frac{5 \gamma}{16} \omega^{2} \tau^{2}+\mathcal{O}\left(\tau^{3}\right)\right) .
$$

IG therefore induces at next-to-leading order a correction to the evolution of the scale factor similar to the case of a negative curvature [26].

As in [12], we consider the present value for the scalar field value consistent with the Solar System constraints:

$$
\gamma \sigma_{0}^{2}=\frac{1}{8 \pi G} \frac{1+8 \gamma}{1+6 \gamma}
$$

where $G=6.67 \times 10^{-8} \mathrm{~cm}^{3} \mathrm{~g}^{-1} \mathrm{~s}^{-2}$ is the gravitational constant measured in laboratory Cavendish-type.

In figure 1 several quantities are plotted versus the scale factor $a$ for $\gamma=10^{-3}$ and for different values of $n$, within the assumption of Eq. 2.10. In the upper left panel, the evolution of $\sigma / \sigma_{0}$ is plotted up to $a=10$ in order to show the dependence on $n$ of the future single field attractor of Eq. 2.6. In the upper right panel we show the parameter of state $w_{\mathrm{DE}}$ of the effective dark energy component corresponding to Einstein gravity with a Newton's constant given by the current value of the scalar field, i.e. $8 \pi G_{\mathrm{N}}=\left(\gamma \sigma_{0}^{2}\right)^{-1}$ as introduced in [18]: also in this case we extend the plot up to $a=10$ to show that $w_{\mathrm{DE}} \simeq-1$ for values of $\gamma$ compatible with observations [12]. In the lower left panel we show the evolution of the critical densities $\Omega_{i}$, always corresponding to an Einstein gravity system with a Newton's constant given by the current value of the scalar field defined in [12]. In the lower right panel, we show explicitly the evolution of $G_{\mathrm{N}}(a) / G \equiv \sigma_{0}^{2} / \sigma^{2}$; it is clear that in this class of models the effective Newton's constant (the effective Planck mass $M_{\mathrm{pl}}^{2}(a)=\gamma \sigma^{2}(a)$ ) decreases (increases) with time.

\section{Linear fluctuations and predictions for cosmological observables}

As in our previous paper for the]] ++ quartic potential [12], we consider scalar fluctuations in the longitudinal gauge. We refer to [12] for the Einstein equations in the longitudinal gauge with the substitution of the quartic potential with a generic monomial one where necessary. The equation for the field fluctuations in the longitudinal gauge for a generic potential is:

$$
\begin{aligned}
\ddot{\delta \sigma}+\dot{\delta \sigma}\left(3 H+2 \frac{\dot{\sigma}}{\sigma}\right)+ & {\left[\frac{k^{2}}{a^{2}}+\left(V_{\sigma \sigma}+4 \frac{V}{\sigma^{2}}-4 \frac{V_{\sigma}}{\sigma}\right)-\frac{\dot{\sigma}^{2}}{\sigma^{2}}+\frac{\sum_{i}\left(\rho_{i}-3 p_{i}\right)}{(1+6 \gamma) \sigma^{2}}\right] \delta \sigma } \\
& =\frac{2 \Psi \sum_{i}\left(\rho_{i}-3 p_{i}\right)}{(1+6 \gamma) \sigma}+\frac{\sum_{i}\left(\delta \rho_{i}-3 \delta p_{i}\right)}{(1+6 \gamma) \sigma}+\dot{\sigma}(3 \dot{\Phi}+\dot{\Psi}) .
\end{aligned}
$$



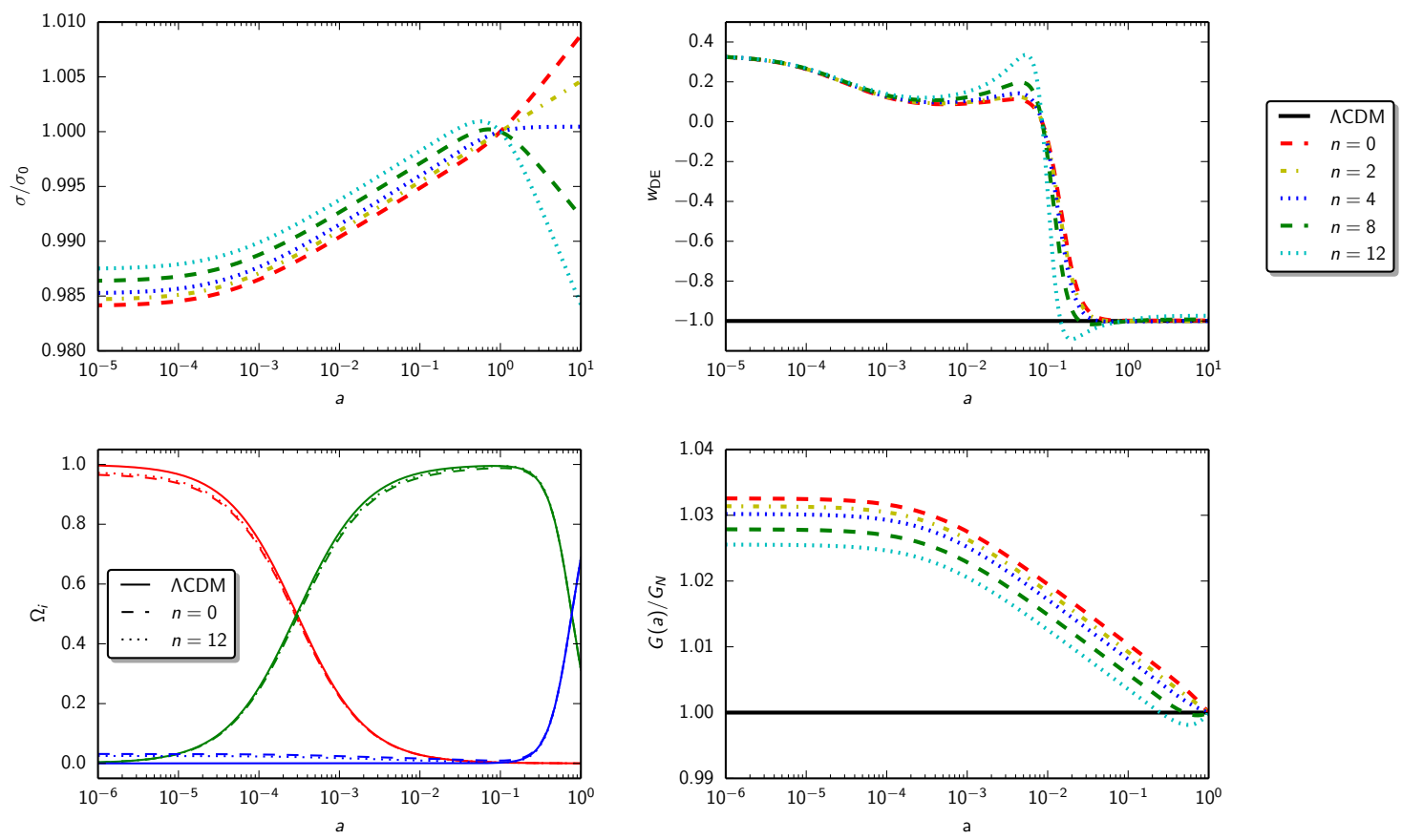

Figure 1. Evolution of $\sigma / \sigma_{0}$ (upper left panel), $w_{\mathrm{DE}}$ (upper right panel) and $G_{\mathrm{N}}(a) / G \equiv \sigma_{0}^{2} / \sigma^{2}$ (lower right panel) as function of the scale factor $a$ for $\gamma=10^{-3}$ and different values of $n$. In the lower left panel we show the critical densities $\Omega_{i}$ : radiation in red, matter in green and effective dark energy in blue. See text for more details.

We note that the terms in the potential and its derivatives in the effective mass of $\delta \sigma$ vanish only for $n=4$. We therefore expect a non-trivial dependence of $\delta \sigma$ on large scales since the onset of accelerated expansion.

We have extended our previous modification [12] of the publicly available EinsteinBoltzmann code CLASS ${ }^{1}[27,28]$ to a generic potential. We therefore test our numerical results obtained by initializing fluctuations in the adiabatic initial conditions deep in the radiation era to the quasi-static approximation beyond the case of a quartic potential, which was studied previously [12]. We consider the parameters $\mu(k, a)$ and $\delta(k, a)$ :

$$
\begin{aligned}
k^{2} \Psi & =-4 \pi G a^{2} \mu(k, a)[\Delta+3(\rho+p) \bar{\sigma}], \\
k^{2}[\Phi-\delta(k, a) \Psi] & =12 \pi G a^{2} \mu(k, a)(\rho+p) \bar{\sigma},
\end{aligned}
$$

where $\Phi, \Psi$ are the Newtonian potentials in the longitudinal gauge, $\Delta$ is the total comoving energy perturbation (excluding the contribution from $\sigma$ ) and $\bar{\sigma}$ is the anisotropic stress [12].

\footnotetext{
${ }^{1}$ www.class-code.net
} 


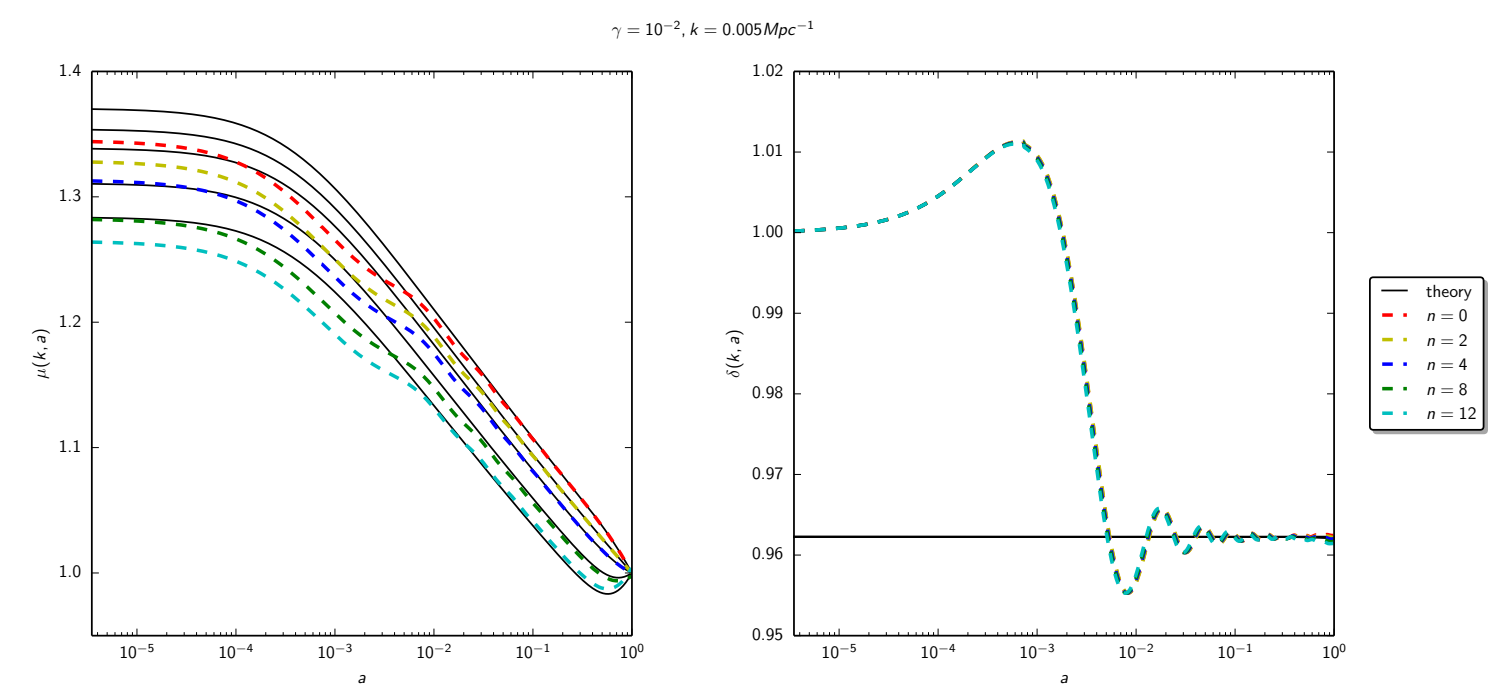

Figure 2. Comparison of the theoretical quasi-static approximations for $\mu(k, a)$ and $\delta(k, a)$ parameters (black lines) with our exact numerical results for $k=0.005 \mathrm{Mpc}^{-1}$ and $\gamma=10^{-2}$ when $n$ is varied.

In the quasi-static approximation, for generic $n$, the two parameters are approximated as:

$$
\begin{aligned}
\mu(k, a) & =\frac{\sigma_{0}^{2}}{\sigma^{2}} \frac{1+6 \gamma}{1+8 \gamma} \frac{1+8 \gamma-2 m_{\mathrm{eff}}^{2} / k^{2}}{1+6 \gamma-2 m_{\mathrm{eff}}^{2} / k^{2}} \\
\delta(k, a) & =\frac{1+4 \gamma-2 m_{\mathrm{eff}}^{2} / k^{2}}{1+8 \gamma-2 m_{\mathrm{eff}}^{2} / k^{2}}
\end{aligned}
$$

where

$$
m_{\mathrm{eff}}^{2}=\frac{d}{d \sigma}\left(\sigma^{4} \frac{d}{d \sigma}\left(\frac{V}{\sigma^{4}}\right)\right) .
$$

Our exact numerical results are compared with the quasi-static approximation in figure 2 for $k=0.005 \mathrm{Mpc}^{-1}$ and $\gamma=10^{-2}$ when $n$ is varied. As already established for the quartic potential [12], the quasi-static approximation for $\mu(k, a)$ is quite accurate only for sub-Hubble scales also in the general case, i.e. $n \neq 4$. The parameter $\delta(k, a)$ depends on time when $n \neq 4$, but depends on $n$ weakly compared to $\mu(k, a)$.

\section{Predictions for CMB anisotropies and Matter Power spectrum}

The power spectra of the CMB temperature anisotropies for different values of $\gamma$ are shown in the left panel of figure 3. The relative differences for CMB temperature anisotropies with respect to a $\Lambda \mathrm{CDM}$ reference model are shown in the right panel of figure 3 for $\gamma=10^{-3}$, $\gamma=10^{-4}$ and in figure 4 for different $n$ and $\gamma=10^{-2}$.

Analogous plots for the E-mode polarization, lensing and linear matter power spectrum (at $z=0$ ) are shown in figures 5, 6, 7 for different $n$ and $\gamma=10^{-2}$. From these plots is clear that the impact of different $n$ is mainly relegated to $\ell \lesssim 30$ in the autocorrelator spectra of 

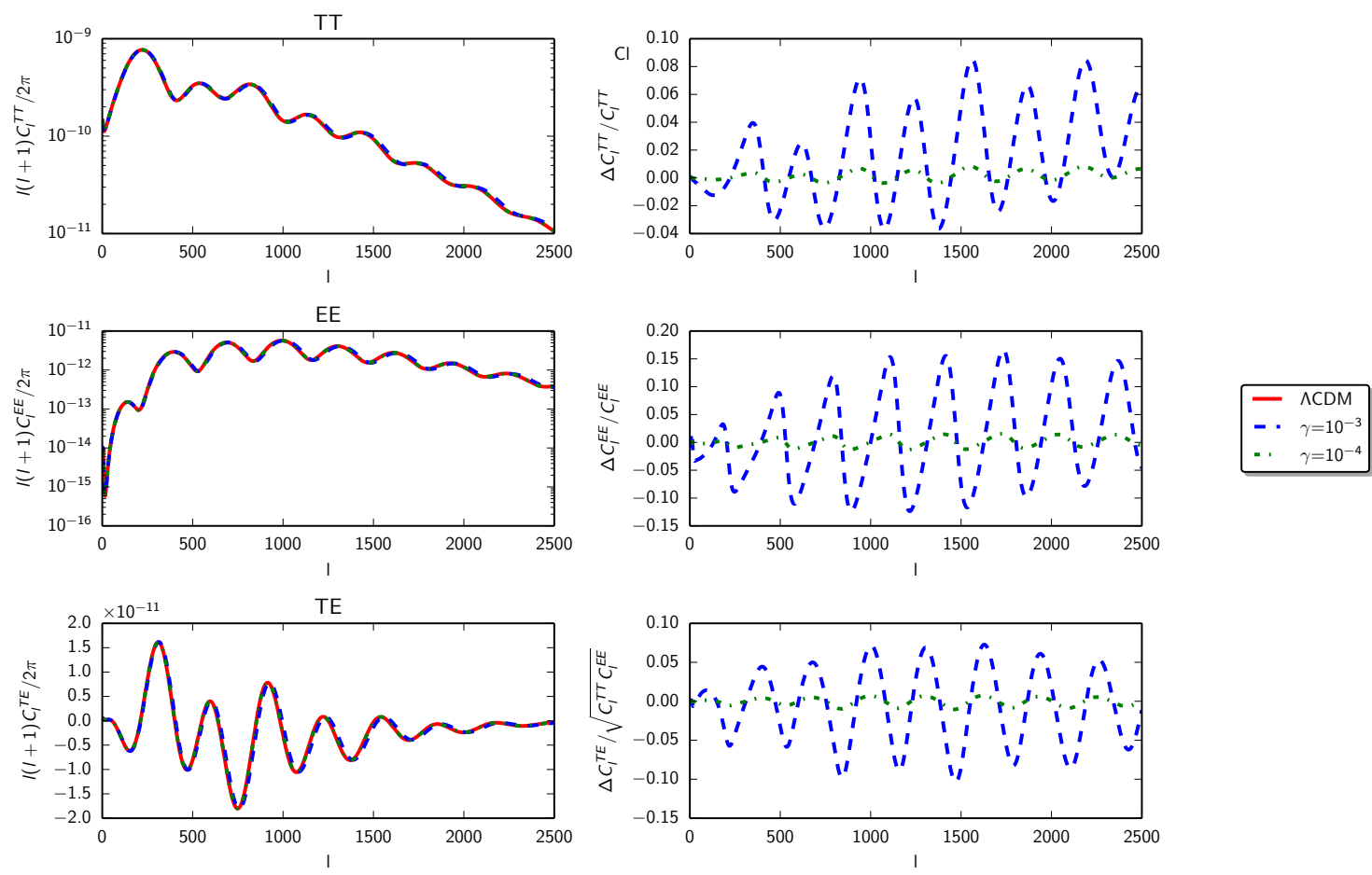

Figure 3. To the left, from the upper to the lower panel respectively, CMB TT, EE and TE power spectra for $\gamma=10^{-3}, 10^{-4}$ and $n=4$. In the upper and middle right panels, we show the relative differences for TT and EE spectra with respect to a reference $\Lambda \mathrm{CDM}$ model. In the lower right panel we show the differences for $C_{\ell}^{T E}$ normalized to $\sqrt{C_{\ell}^{T T} C_{\ell}^{E E}}$.

CMB temperature and polarization. The impact of different potentials is not negligible at smaller scales. Instead, is not the case for CMB lensing (figure 6) and the linear matter power spectrum (figure 7).

\section{Updated Planck 2015 results for the quartic potential}

In this and the following section we constrain this simple class of dark energy models with the Planck $[1,5,20]$ and a compilation of BAO [21-23] data.

We performed the Markov Chain Monte Carlo (MCMC) analysis by using the publicly available code Monte PYTHON ${ }^{2}$ [29] connected to our modified version of the code CLASS. We varied the six cosmological parameters for a flat $\Lambda$ CDM model, i.e. $\omega_{b}, \omega_{c}, H_{0}, \tau$, $\ln \left(10^{10} A_{s}\right), n_{s}$, plus one extra parameter related to the coupling with the Ricci curvature in Eq. (2.1). We sampled on the quantity $\zeta$, according to [12, 30], defined as:

$$
\zeta \equiv \ln (1+4 \gamma)=\ln \left(1+\frac{1}{\omega_{\mathrm{BD}}}\right)
$$

with the prior $[0,0.039]$. In the analysis we assumed 3 massless neutrino and marginalized over foreground and calibration nuisance parameters which are also varied together with the cosmology.

\footnotetext{
${ }^{2}$ https://github.com/baudren/montepython_public
} 

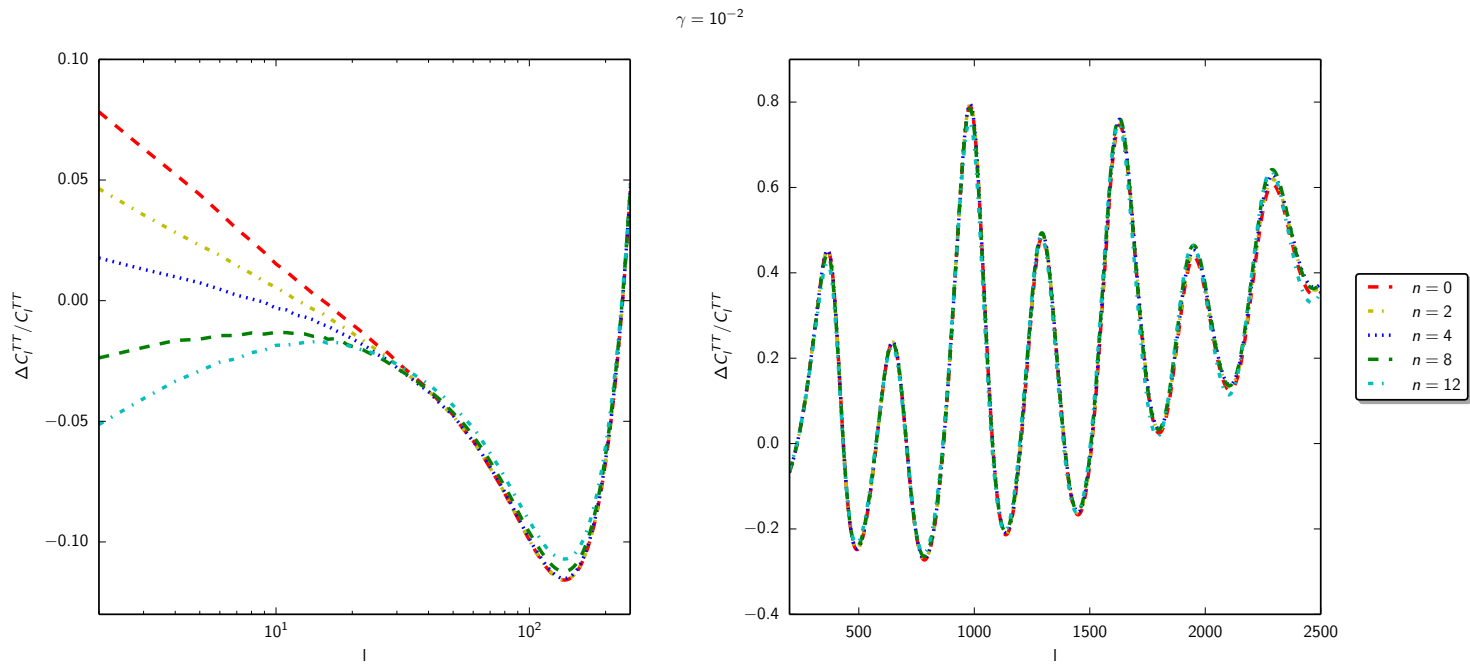

Figure 4. Relative differences for the CMB temperature anisotropies power spectrum with respect to a reference $\Lambda \mathrm{CDM}$ for $\gamma=10^{-2}$ and different values of $n$ are shown for $\ell<300$ (left panel) and for $\ell>200$ (right panel).
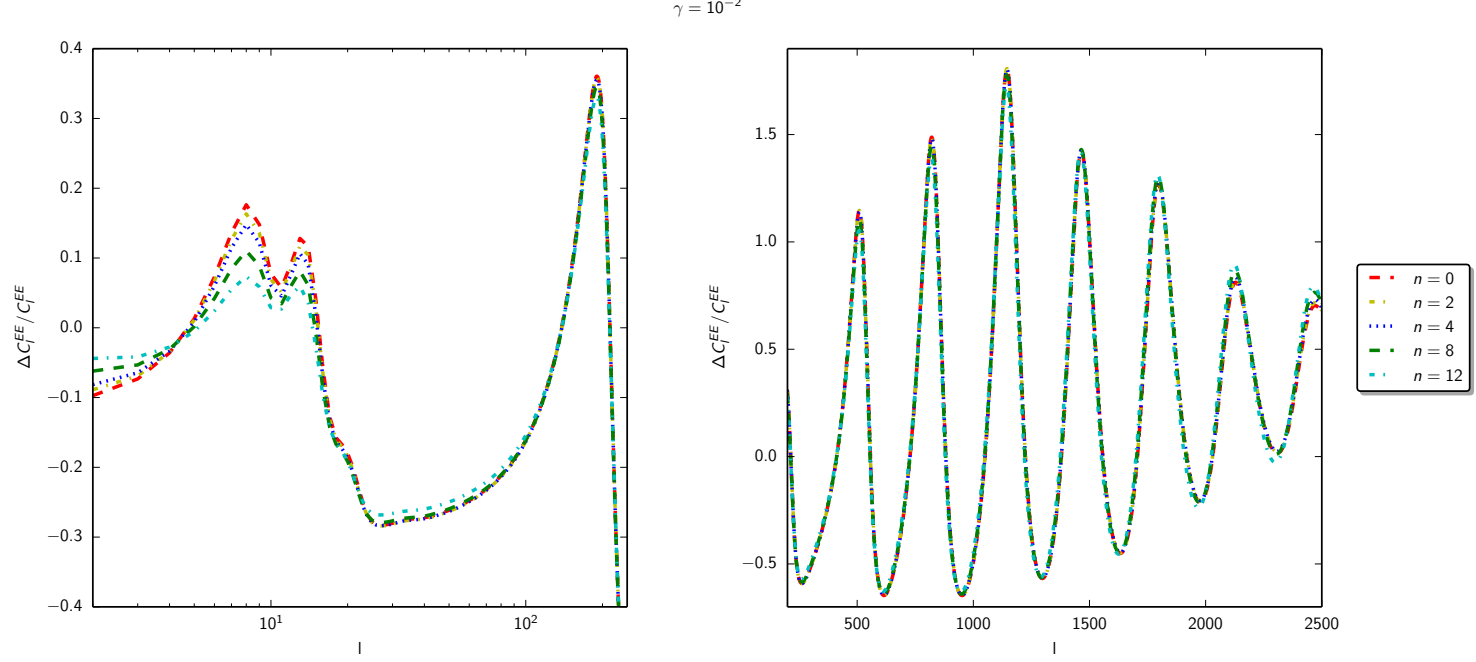

Figure 5. Relative differences for the CMB polarization E-mode anisotropies power spectrum with respect to a reference $\Lambda \mathrm{CDM}$ for $\gamma=10^{-2}$ and different values of $n$ are shown for $\ell<300$ (left panel) and for $\ell>200$ (right panel).

As CMB data we use the Planck 2015 release [20] based on the two-point function as provided by 1 . an exact pixel based likelihood at low resolution, which covers temperature and polarization data from $\ell=2$ to 29 (the polarization part of this likelihood is denoted as "lowP" in the following), 2. a high- $\ell$ likelihood based on a Gaussian approximation available as temperature only or temperature plus polarization. We will refer in the following to Planck TT (TT,TE,EE) as the combination of the TT (TT,TE,EE) likelihood at multipoles $\ell \geq 30$ and the low- $\ell$ temperature-only likelihood. We also use the Planck 2015 lensing likelihood [5], in particular the version obtained from temperature and polarization data, with the multipole 

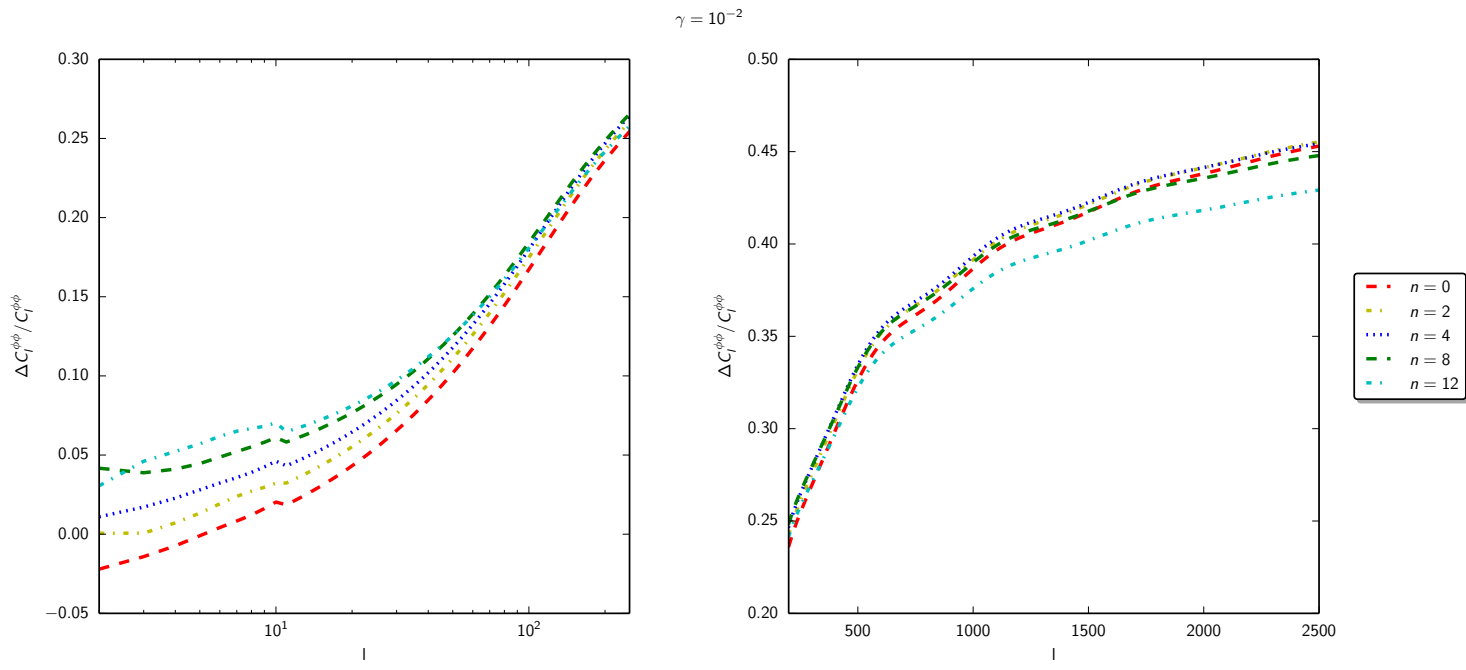

Figure 6. Relative differences for the CMB lensing power spectrum with respect to a reference $\Lambda \mathrm{CDM}$ for $\gamma=10^{-2}$ and different values of $n$ are shown for $\ell<300$ (left panel) and for $\ell>200$ (right panel).
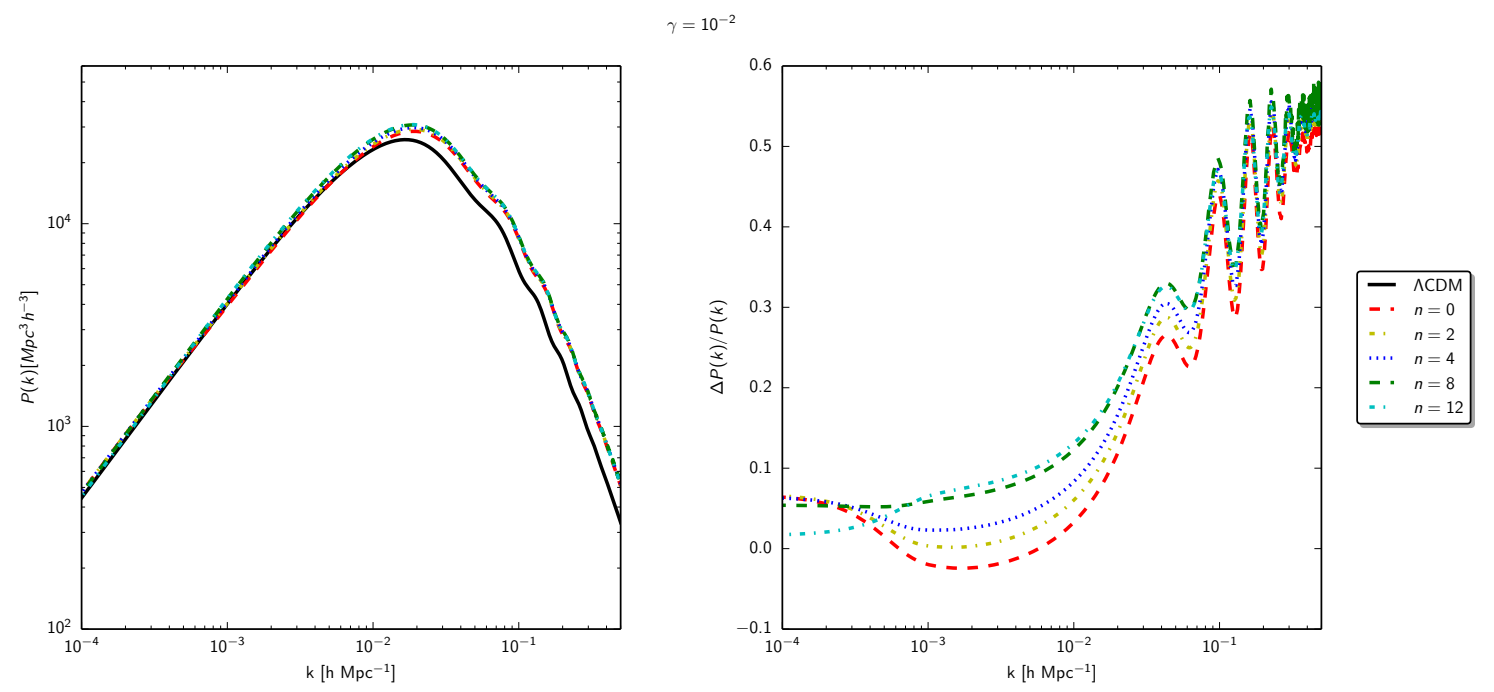

Figure 7. In the left panel, we show the linear matter power spectrum for $\gamma=10^{-2}$ and different values of $n$. In the right panel, we show the relative difference for the linear matter power spectrum with respect to a reference $\Lambda \mathrm{CDM}$.

range $40 \leq \ell \leq 400$.

In combination with CMB data we use measurments of $D_{V} / r_{\text {drag }}$ by 6 dFGRS at $z_{\text {eff }}=$ 0.106 [21], SDSS-MGS at $z_{\mathrm{eff}}=0.15$ [22], SDSS-DR11 CMASS and LOWZ at $z_{\mathrm{eff}}=0.57$ and $z_{\text {eff }}=0.32$ respectively [23]. Moreover, in section 5.1 we consider the impact of the local measurements on the Hubble's constant, i.e. $H_{0}=73.8 \pm 2.4 \mathrm{~km} \mathrm{~s}^{-1} \mathrm{Mpc}^{-1}$ [32] and $H_{0}=70.6 \pm 3.0 \mathrm{~km} \mathrm{~s}^{-1} \mathrm{Mpc}^{-1}$ [33].

The constraints obtained from $\mathrm{CMB}$ and $\mathrm{BAO}$ data with $n=4$, for different combinations of data sets are summarized in table 1. These results update those presented in 

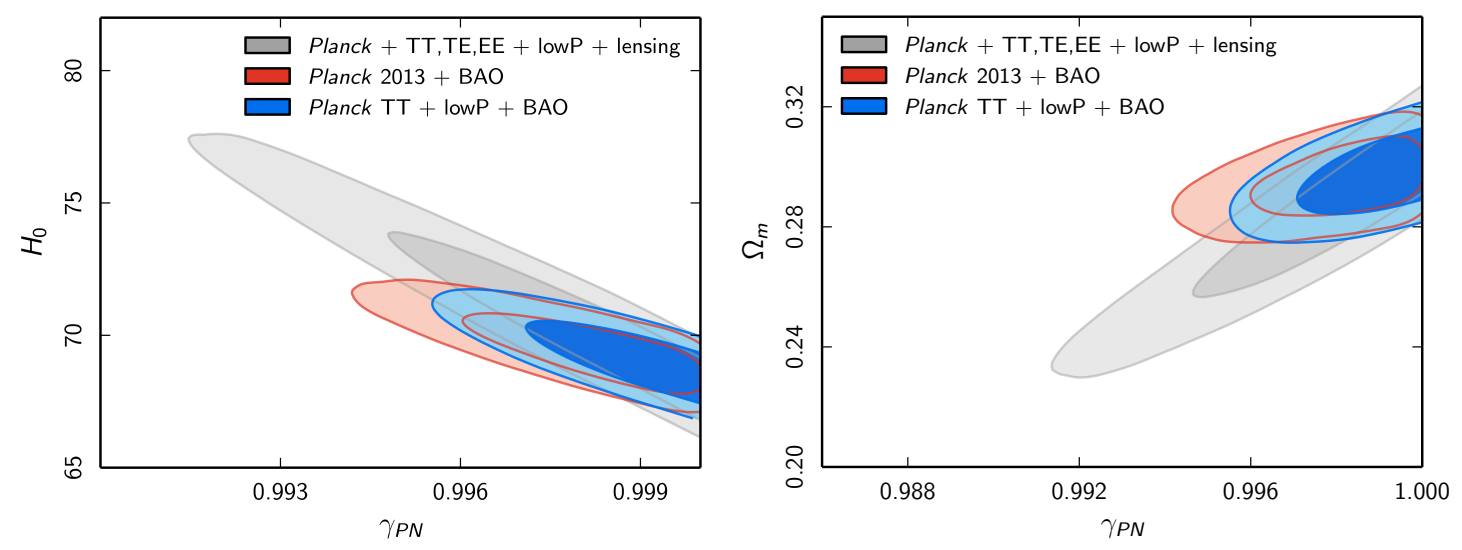

Figure 8. 2-dimentional marginalized confidence levels at $68 \%$ and $95 \%$ for $\left(\gamma_{\mathrm{PN}}, H_{0}\right)$ on the left and $\left(\gamma_{\mathrm{PN}}, \Omega_{\mathrm{m}}\right)$ on the right for Planck TT,TE,EE + lowP + lensing (gray), Planck $2013+\mathrm{BAO}$ (red) and Planck TT + lowP + BAO (blue).
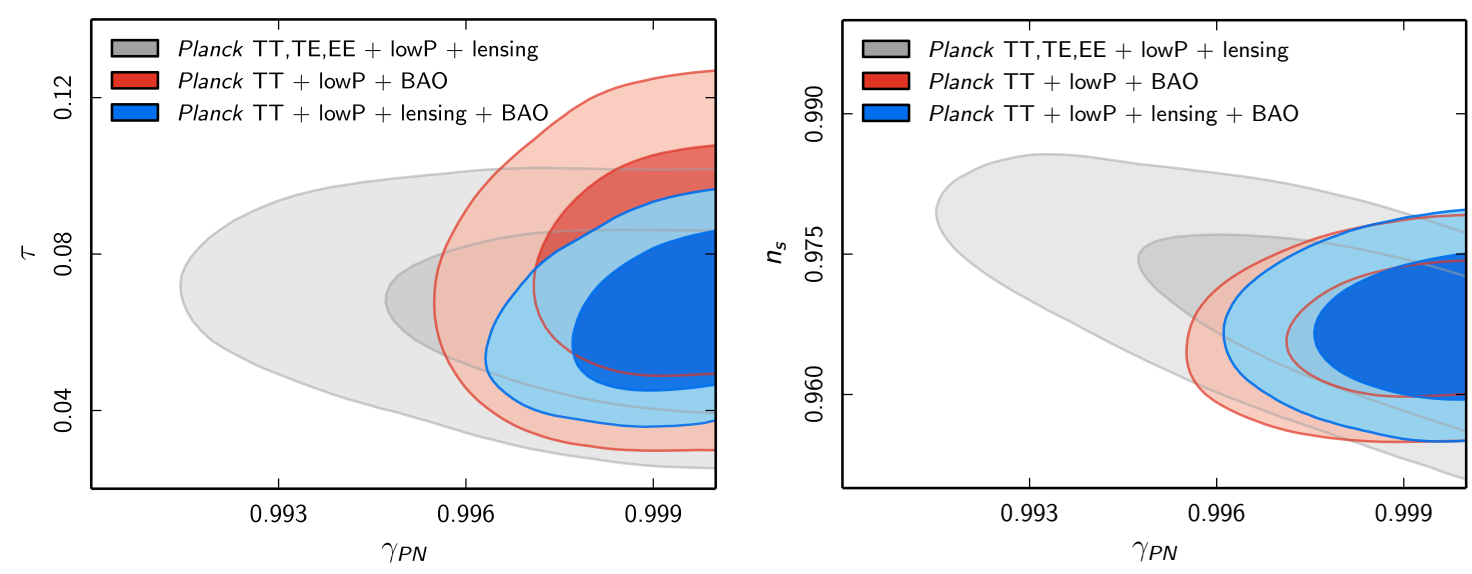

Figure 9. 2-dimentional marginalized confidence levels at $68 \%$ and $95 \%$ for $\left(\gamma_{\mathrm{PN}}, \tau\right)$ on the left and $\left(\gamma_{\mathrm{PN}}, n_{\mathrm{s}}\right)$ on the right for Planck TT,TE,EE + lowP + lensing (gray), Planck $2013+$ BAO (red) and Planck TT + lowP + BAO (blue).

Ref. [12] based on the Planck nominal mission temperature data, and use the same compilation of BAO data. See also [30, 34, 35] for other works studying Planck 2013 constraints on Brans-Dicke-like models. In combination the same BAO data, the full mission temperature data improve the $95 \%$ CL constraint on the coupling to the Ricci curvature $\gamma$ by $25 \%$ compared to the nominal mission data (see also figure 8) to:

$$
\gamma<0.00089 \text { (95\% CL, Planck TT }+ \text { lowP }+ \text { BAO) } .
$$

We now discuss the impact of the Planck lensing data [5]. One of the effects of the CMB lensing is to slightly favour smaller values of the amplitude of fluctuations $A_{\mathrm{s}}$ and therefore of the optical depth thanks to the accurate determination of $A_{\mathrm{s}} e^{-2 \tau}$ by the CMB temperature power spectrum measured by PLANCK. We show in figure 9 how the addition of Planck lensing improves either the determination of $\tau$ and the constraint on $\gamma$. 


\begin{tabular}{|l|ccc|}
\hline & $\begin{array}{c}\text { Planck } 2013 \\
+\mathrm{BAO}\end{array}$ & $\begin{array}{c}\text { Planck TT }+ \text { lowP } \\
+\mathrm{BAO}\end{array}$ & $\begin{array}{c}\text { Planck TT }+ \text { lowP } \\
+ \text { lensing }+\mathrm{BAO}\end{array}$ \\
\hline $10^{5} \Omega_{\mathrm{b}} h^{2}$ & $2203 \pm 25$ & $222 \pm 21$ & $2224_{-21}^{+20}$ \\
$10^{4} \Omega_{\mathrm{C}} h^{2}$ & $1207_{-22}^{+18}$ & $1198_{-17}^{+16}$ & $1191 \pm 14$ \\
$H_{0}\left[\mathrm{~km} \mathrm{~s}^{-1} \mathrm{Mpc}^{-1}\right]$ & $69.5_{-1.2}^{+0.9}$ & $69.4_{-1.0}^{+0.8}$ & $69.4_{-0.9}^{+0.7}$ \\
$\tau$ & $0.088_{-0.013}^{+0.012}$ & $0.076_{-0.018}^{+0.019}$ & $0.063_{-0.014}^{+0.012}$ \\
$\ln \left(10^{10} A_{\mathrm{s}}\right)$ & $3.090_{-0.024}^{+0.024}$ & $3.087 \pm 0.036$ & $3.059_{-0.022}^{+0.022}$ \\
$n_{\mathrm{S}}$ & $0.9611 \pm 0.0053$ & $0.9665 \pm 0.0046$ & $0.9669_{-0.0047}^{-0.0042}$ \\
$\zeta$ & $<0.0047(95 \% \mathrm{CL})$ & $<0.0036(95 \% \mathrm{CL})$ & $<0.0031(95 \% \mathrm{CL})$ \\
\hline $10^{3} \gamma$ & $<1.2(95 \% \mathrm{CL})$ & $<0.89(95 \% \mathrm{CL})$ & $<0.75(95 \% \mathrm{CL})$ \\
$\gamma_{P N}$ & $>0.9953(95 \% \mathrm{CL})$ & $>0.9965(95 \% \mathrm{CL})$ & $>0.9970(95 \% \mathrm{CL})$ \\
$\Omega_{\mathrm{m}}$ & $0.295 \pm 0.009$ & $0.295 \pm 0.008$ & $0.294 \pm 0.008$ \\
$\delta G_{\mathrm{N}} / G_{\mathrm{N}}$ & $-0.015_{-0.006}^{+0.013}$ & $-0.011_{-0.004}^{+0.010}$ & $-0.009_{-0.009}^{+0.003}$ \\
$10^{13} \dot{G}_{\mathrm{N}}(z=0) / G_{\mathrm{N}}\left[\mathrm{yr}^{-1}\right]$ & $-0.61_{-0.25}^{+0.55}$ & $-0.45_{-0.16}^{+0.43}$ & $-0.37_{-0.12}^{+0.34}$ \\
$10^{23} \ddot{G}_{\mathrm{N}}(z=0) / G_{\mathrm{N}}\left[\mathrm{yr}^{-2}\right]$ & $0.86_{-0.78}^{+0.33}$ & $0.63_{-0.58}^{+0.22}$ & $0.52_{-0.50}^{+0.17}$ \\
\hline
\end{tabular}

Table 1. Constraints on main and derived parameters for Planck TT + lowP + BAO (at $68 \%$ CL if not otherwise stated). In the first column we report the results obtained with the previous Planck 2013 data from Ref. [12]

\begin{tabular}{|l|ccc|}
\hline & $\begin{array}{c}\text { Planck TT,TE,EE } \\
\text { + lowP + lensing }\end{array}$ & $\begin{array}{c}\text { Planck TT,TE,EE } \\
\text { + lowP }+ \text { BAO }\end{array}$ & $\begin{array}{c}\text { Planck TT, TE,EE } \\
\text { + lowP + lensing + BAO }\end{array}$ \\
\hline $10^{5} \Omega_{\mathrm{b}} h^{2}$ & $2234 \pm 17$ & $2231 \pm 14$ & $2223 \pm 20$ \\
$10^{4} \Omega_{\mathrm{c}} h^{2}$ & $1189 \pm 14$ & $1194 \pm 12$ & $1191_{-14}^{+15}$ \\
$H_{0}\left[\mathrm{~km} \mathrm{~s}^{-1} \mathrm{Mpc}^{-1}\right]$ & $71.0_{-3.0}^{+1.4}$ & $69.4_{-1.1}^{+0.6}$ & $69.4_{-1.0}^{+0.5}$ \\
$\tau$ & $0.066_{-0.013}^{+0.012}$ & $0.079_{-0.016}^{+0.017}$ & $0.063_{-0.014}^{+0.012}$ \\
$\ln \left(10^{10} A_{\mathrm{s}}\right)$ & $3.066_{-0.028}^{+0.024}$ & $3.095_{-0.033}^{+0.031}$ & $3.059_{-0.026}^{+0.021}$ \\
$n_{\mathrm{s}}$ & $0.9695 \pm 0.0056$ & $0.9675 \pm 0.0041$ & $0.9669_{-0.0043}^{-0.0043}$ \\
$\zeta$ & $<0.0068(95 \% \mathrm{CL})$ & $<0.0030(95 \% \mathrm{CL})$ & $<0.0030(95 \% \mathrm{CL})$ \\
\hline $10^{3} \gamma$ & $<1.7(95 \% \mathrm{CL})$ & $<0.76(95 \% \mathrm{CL})$ & $<0.75(95 \% \mathrm{CL})$ \\
$\gamma_{P N}$ & $>0.9933(95 \% \mathrm{CL})$ & $>0.9970(95 \% \mathrm{CL})$ & $>9970(95 \% \mathrm{CL})$ \\
$\Omega_{\mathrm{m}}$ & $0.281 \pm 0.009$ & $0.295 \pm 0.015$ & $0.294 \pm 0.008$ \\
$\delta G_{\mathrm{N}} / G_{\mathrm{N}}$ & $-0.020_{-0.005}^{+0.019}$ & $-0.010_{-0.009}^{+0.004}$ & $-0.009_{-0.009}^{+0.003}$ \\
$10^{13} \dot{G}_{\mathrm{N}}(z=0) / G_{\mathrm{N}}\left[\mathrm{yr}^{-1}\right]$ & $-0.77_{-0.27}^{+0.43}$ & $-0.39_{-0.15}^{+0.35}$ & $-0.37_{-0.12}^{+0.34}$ \\
$10^{23} \ddot{G}_{\mathrm{N}}(z=0) / G_{\mathrm{N}}\left[\mathrm{yr}^{-2}\right]$ & $1.2_{-1.1}^{+0.4}$ & $0.56_{-0.50}^{+0.21}$ & $0.52_{-0.50}^{+0.17}$ \\
\hline
\end{tabular}

Table 2. Constraints on main and derived parameters for Planck TT,TE,EE + lowP with different combination of other datasets (at $68 \% \mathrm{CL}$ if not otherwise stated).

In table 2 we show the results with the inclusion of Planck high- $\ell$ polarization data. The IG dark energy model with a quartic potential provide a better fit of the data compared to $\Lambda \mathrm{CDM}$, but not at a statistically significant level $-\Delta \chi^{2} \simeq-1.2$ for Planck $\mathrm{TT}+$ lowP $+\mathrm{BAO}$ and $\Delta \chi^{2} \simeq-2.3$ for Planck TT,TE,EE + lowP + lensing. It is important to note that the full information of PLANCK alone, i.e. temperature, polarization and lensing, is now capable to constrain $\gamma$ :

$$
\gamma<0.0017 \text { (95\% CL, Planck TT,TE,EE + lowP + lensing). }
$$

We quote the following Planck TT,TE,EE + lowP + lensing + BAO at the $95 \%$ CL constraint on the coupling to the Ricci curvature:

$$
\gamma<0.00075 \text { (95\% CL, Planck TT,TE,EE + lowP + lensing + BAO) . }
$$

We quote also the derived constraints on the change of the effective Newton's constant between the radiation era and the present time $\delta G_{\mathrm{N}} / G_{\mathrm{N}} \equiv\left(\sigma_{i}^{2}-\sigma_{0}^{2}\right) / \sigma_{0}^{2}$ :

$$
\frac{\delta G_{\mathrm{N}}}{G_{\mathrm{N}}}=-0.002_{-0.037}^{+0.002}(95 \% \mathrm{CL}, \text { Planck TT,TE,EE }+ \text { lowP }+\mathrm{BAO})
$$


and the constraint on its derivatives $\left(\dot{G}_{\mathrm{N}} / G_{\mathrm{N}} \equiv-2 \dot{\sigma}_{0} / \sigma_{0}\right)$ at present time:

$$
\begin{aligned}
& \frac{\dot{G}_{\mathrm{N}}}{G_{\mathrm{N}}}(z=0)=-0.08_{-0.55}^{+0.08}\left[\times 10^{-13} \mathrm{yr}^{-1}\right](95 \% \mathrm{CL}, \text { Planck TT,TE,EE }+ \text { lowP }+ \text { BAO }), \\
& \frac{\ddot{G}_{\mathrm{N}}}{G_{\mathrm{N}}}(z=0)=0.36_{-0.36}^{+0.26}\left[\times 10^{-23} \mathrm{yr}^{-1}\right](95 \% \mathrm{CL}, \text { Planck TT,TE,EE }+ \text { lowP }+\mathrm{BAO}) .
\end{aligned}
$$

The constraints derived here are the tighter obtained from cosmological observations for similar scalar-tensor models (for a comparison, the $95 \%$ CL constraint from pulsar timing is $\left.\dot{G}_{\mathrm{N}} / G_{\mathrm{N}}=-0.6 \pm 1.1 \times 10^{-12} \mathrm{yr}^{-1}[31]\right)$.

\subsection{Combination with local measurements}

As in [12] we analyze the combination of the local measurements of the Hubble constant with Planck TT + lowP by considering the impact of two different local estimates of $H_{0}$, such as: $H_{0}=73.8 \pm 2.4 \mathrm{~km} \mathrm{~s}^{-1} \mathrm{Mpc}^{-1}$ [32], denoted as $H_{0}^{*}$, and $H_{0}=70.6 \pm 3.0 \mathrm{~km} \mathrm{~s}^{-1} \mathrm{Mpc}^{-1}$ [33], denoted as $H_{0}^{\dagger}$. We find:

$$
\begin{aligned}
& H_{0}=73.1_{-2.3}^{+2.1}\left[\mathrm{~km} \mathrm{~s}^{-1} \mathrm{Mpc}^{-1}\right]\left(68 \% \mathrm{CL}, \text { Planck } \mathrm{TT}+\text { lowP }+\mathrm{H}_{0}^{*}\right) \\
& \gamma=0.0011 \pm 0.0010\left(95 \% \mathrm{CL}, \text { Planck } \mathrm{TT}+\text { lowP }+\mathrm{H}_{0}^{*}\right),
\end{aligned}
$$

and

$$
\begin{aligned}
& H_{0}=71.3_{-2.8}^{+1.8}\left[\mathrm{~km} \mathrm{~s}^{-1} \mathrm{Mpc}^{-1}\right]\left(68 \% \mathrm{CL}, \text { Planck } \mathrm{TT}+\text { lowP }+\mathrm{H}_{0}^{\dagger}\right) \\
& \gamma<0.0017\left(95 \% \mathrm{CL}, \text { Planck } \mathrm{TT}+\text { lowP }+\mathrm{H}_{0}^{\dagger}\right) .
\end{aligned}
$$

We note that the degeneracy of $H_{0}$ with higher value of $\gamma$ has been reduced with the improved accuracy of the Planck full mission temperature data, compared to the nominal mission data [12].

In combination with $\mathrm{BAO}$, we find:

$$
H_{0}=69.4_{-1.0}^{+0.8}\left[\mathrm{~km} \mathrm{~s}^{-1} \mathrm{Mpc}^{-1}\right](68 \% \mathrm{CL}, \text { Planck TT }+ \text { lowP }+\mathrm{BAO}),
$$

which is larger than the value obtained for the $\Lambda \mathrm{CDM}$ model with three massless neutrinos, i.e. $67.8 \pm 0.6 \mathrm{~km} \mathrm{~s}^{-1} \mathrm{Mpc}^{-1}$, for the same combination of datasets.

\section{2 $\quad$ BBN consistency relation on $G_{\mathrm{N}}$}

The value of the effective gravitational constant determines the expansion rate in the radiation era and therefore can affect the cosmological abundances of the light elements during Big Bang Nucleosynthesis (BBN). Therefore, BBN was used to provide limits to the variation of the effective Newton's constant [36, 37].

In the following we investigate the impact of the modification of the BBN consistency condition implemented in the public code PARTHENOPE [38] due to the different value of the effective Newton's constant during nucleosynthesis. We consider the effect of a different gravitational constant as a source of extra radiation in $Y_{\mathrm{P}}^{\mathrm{BBN}}\left(\omega_{b}, N_{\text {eff }}\right)$, [39]. It is interesting to note that with this improved BBN consistency condition the posterior probabilities for the primary cosmological parameters are unaffected, and we just observe a small shift for the primordial Helium abundance towards higher values. 


\begin{tabular}{|l|ccccc|}
\hline & $n=0$ & $n=2$ & $n=4$ & $n=6$ & $n=8$ \\
\hline$\delta G_{\mathrm{N}} / G_{\mathrm{N}}$ & $>-0.028$ & $>-0.027$ & $>-0.026$ & $>-0.026$ & $>-0.025$ \\
$10^{13} \dot{G}_{\mathrm{N}}(z=0) / G_{\mathrm{N}}\left[\mathrm{yr}^{-1}\right]$ & $>-3.9$ & $>-2.5$ & $>-0.11$ & $<0.4$ & $<1.9$ \\
$10^{23} \ddot{G}_{\mathrm{N}}(z=0) / G_{\mathrm{N}}\left[\mathrm{yr}^{-2}\right]$ & $<0.077$ & $<0.78$ & $<1.5$ & $<2.3$ & $<3.0$ \\
\hline
\end{tabular}

Table 3. Constraints on the variation of the gravitational constant and its time derivatives (at $95 \%$ CL) for different values of $n$ with Planck $\mathrm{TT}+$ lowP $+\mathrm{BAO}$

\section{Cosmological constraints for $n \neq 4$}

In this section we explore the impact of different $n$ on the cosmological parameters. Current data cannot discriminate at a statistical significant level between different values of $n$ and we therefore fix $n$ to representative values $(n=0,2,6,8)$ and we vary the seven primary cosmological parameters (as well as the other foreground/nuisance parameters) as done in the previous section. Figure 10 shows that the posterior probabilities for the seven primary cosmological parameters hardly change for these different values of $n$ : these results are compatible with the dependence of CMB anisotropies on $n$ shown in figures 3,5 . Therefore, this class of scalar-tensor models is compatible with current cosmological data for a larger value of $H_{0}$ and a slightly smaller value for $\Omega_{\mathrm{m}}$.

On the opposite, we note that the posterior probabilities for $\dot{G}_{\mathrm{N}} / G_{\mathrm{N}}$ and $\ddot{G}_{\mathrm{N}} / G_{\mathrm{N}}$ at present time depend on $n$. The dependence of the bounds $\dot{G}_{\mathrm{N}} / G_{\mathrm{N}}$ on $n$ can be easily understood from Eq. 3: for $n=6$ and $n=8$, the derivative of the scalar field becomes negative at the onset of the accelerated stage. This dependence on $n$ must be kept in mind when comparing cosmological bounds on $\dot{G}_{\mathrm{N}} / G_{\mathrm{N}}(z=0)$ and $\ddot{G}_{\mathrm{N}} / G_{\mathrm{N}}(z=0)$ (which depend on the form of the potential) with Solar System constraints on the same time variations (which are obtained extrapolating from the massless case since the effect of the potential is considered negligible on such smaller scales detached from the cosmological expansion).

The bound on the shift of the scalar field between today and the radiation era is the same however it's evolution show a strong dependence from the choice of the potential as summarized in table 3.

\section{Conclusions}

We have studied a simple class of modified gravity models alternative to $\Lambda \mathrm{CDM}$, based on IG - or a Brans-Dicke-like - with a monomial potential. We have limited ourselves to positive values of the exponent, extending the case of a quartic potential previously studied in [12]. In this class of models the scalar field increases from the constant value during the radiation era and therefore the effective Planck mass therefore increase in time during the matter dominated era.

Despite its semplicity, this class of models leads to distinct effects compared to $\Lambda$ CDM for values of the coupling $\gamma$ compatible with observations, such as a slightly larger value of $H_{0}$ because of the modification of the expansion history due to the coupling of the scalar field to the Ricci curvature $\gamma$. We have shown that the latter effect causes a shift of the CMB peaks similar to an open Universe. The dependence on the potential which drives the Universe in acceleration instead reveals itself in the Integrated Sachs-Wolfe effect, i.e. at $\ell \lesssim 20$, for CMB and in general at low redshifts for other cosmological probes, although to a much smaller extent. We have shown that the posterior probabilities for the standard cosmological parameters obtained with the most recent cosmological data are unchanged for 

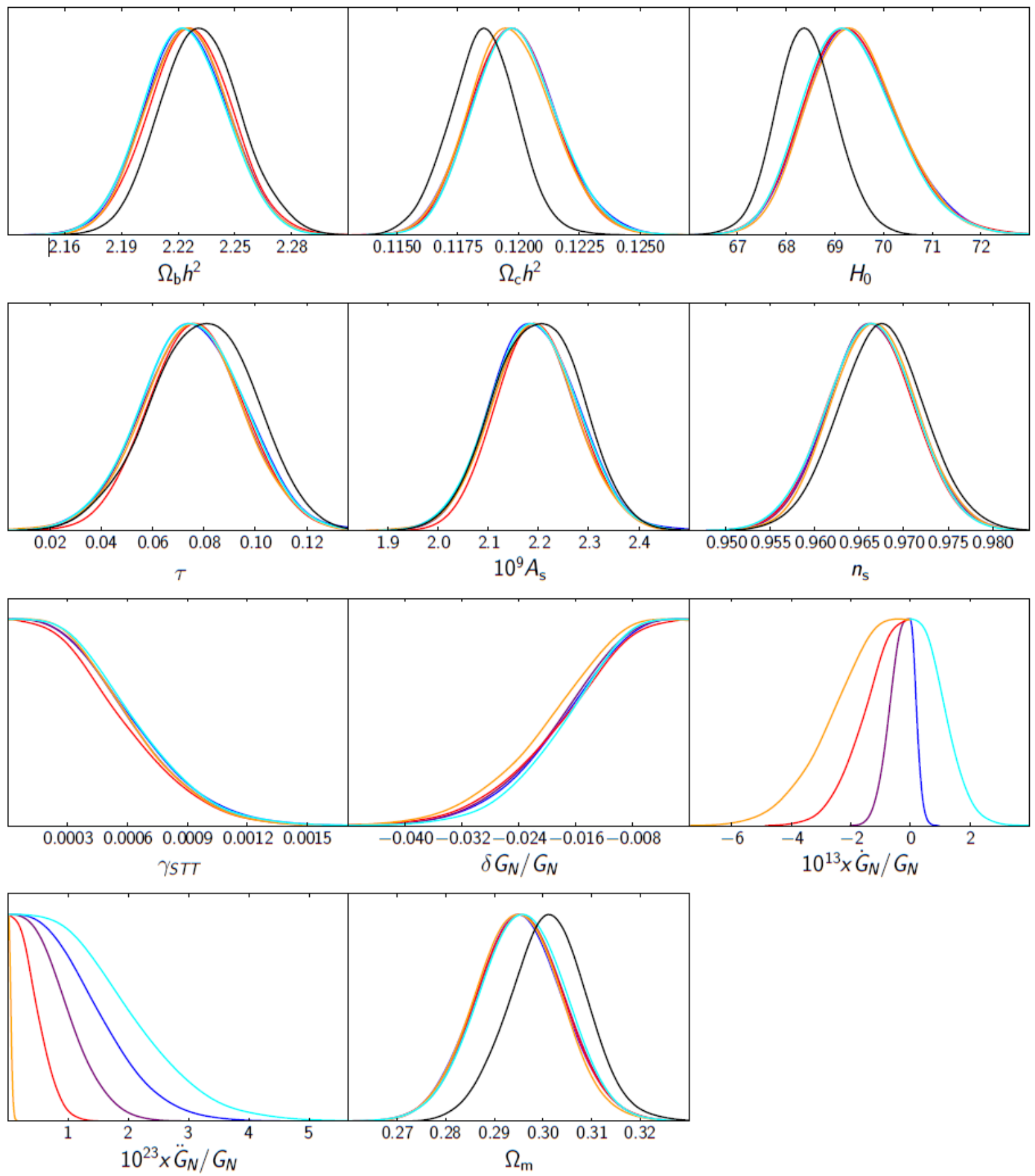

Figure 10. 1-dimentional likelihoods for the cosmological parameters for IG with different index of the monomial potential with Planck TT + BAO, i.e. $\mathrm{n}=0$ (orange), 2 (red), 4 (purple), 6 (blue) and 8 (cyan). We show the comparison w.r.t. the $\Lambda$ CDM cosmologigy plotted in black.

different powers of the monomial potential. On the opposite, current values of $\dot{G} / G$ and $\ddot{G} / G$ (on cosmological scales) depend on the type of the potential: this dependence must be taken in mind when comparing the cosmological constraints with the Solar System constraints. 
The full information of PLANCK alone, i.e. temperature, polarization and lensing, is now capable to constrain $\gamma<0.0017$ at $95 \%$ CL for $n=4$; by adding a compilation of BAO data this $95 \%$ CL constrant is further tightened to $\gamma<0.00075$. With the increasing precision of cosmological observations the cosmological bounds on the variation of $G$ are fully consistent and closer to the Solar System constraints [40].

Future accurate measurments from the Euclid ESA mission [41, 42] will further constrain $\gamma$ and will provide insights on the form of the potential in these induced gravity - or BransDicke-like - dark energy models.

\section{Acknowledgements}

We wish to thank Julien Lesgourgues and Thomas Tram for many useful suggestions on the use of the CLASS code and Benjamin Audren for the useful suggestions on the use of the MONTEPYTHON code. We wish to thank Jan Hamann for useful discussions on Big Bang nucleosynthesis. The support by the "ASI/INAF Agreement 2014-024-R.0 for the Planck LFI Activity of Phase E2" is acknowledged. We acknowledge financial contribution from the agreement ASI/INAF n. I/023/12/1. MB acknowledges the TTK at RWTH University of Aachen for hospitality during writing part of this paper. CU has been supported within the Labex ILP (reference ANR-10-LABX-63) part of the Idex SUPER, and received financial state aid managed by the Agence Nationale de la Recherche, as part of the programme Investissements d'avenir under the reference ANR-11-IDEX-0004-02.

\section{References}

[1] R. Adam et al. [Planck Collaboration], arXiv:1502.01582 [astro-ph.CO].

[2] P. A. R. Ade et al. [Planck Collaboration], arXiv:1502.01589 [astro-ph.CO].

[3] P. A. R. Ade et al. [Planck Collaboration], arXiv:1502.01590 [astro-ph.CO].

[4] G. B. Zhao, H. Li, E. V. Linder, K. Koyama, D. J. Bacon and X. Zhang, Phys. Rev. D 85 (2012) 123546.

[5] P. A. R. Ade et al. [Planck Collaboration], arXiv:1502.01591 [astro-ph.CO].

[6] E. Di Valentino, A. Melchiorri and J. Silk, arXiv:1509.07501 [astro-ph.CO].

[7] A. Zee, Phys. Rev. Lett. 44 (1980) 703.

[8] C. Brans and R. H. Dicke, Phys. Rev. 124 (1961) 925.

[9] F. Cooper and G. Venturi, Phys. Rev. D 24 (1981) 3338.

[10] C. Wetterich, Nucl. Phys. B 302 (1988) 668.

[11] F. Finelli, A. Tronconi and G. Venturi, Phys. Lett. B 659 (2008) 466.

[12] C. Umiltà, M. Ballardini, F. Finelli and D. Paoletti, JCAP 1508 (2015) 08, 017.

[13] J. P. Uzan, Phys. Rev. D 59 (1999) 123510

[14] F. Perrotta, C. Baccigalupi and S. Matarrese, Phys. Rev. D 61 (1999) 023507.

[15] N. Bartolo and M. Pietroni, Phys. Rev. D 61 (2000) 023518

[16] L. Amendola, Phys. Rev. D 60 (1999) 043501

[17] T. Chiba, Phys. Rev. D 60 (1999) 083508.

[18] B. Boisseau, G. Esposito-Farese, D. Polarski and A. A. Starobinsky, Phys. Rev. Lett. 85 (2000) 2236. 
[19] T. Chiba, M. Siino and M. Yamaguchi, Phys. Rev. D 81 (2010) 083530.

[20] N. Aghanim et al. [Planck Collaboration], arXiv:1507.02704 [astro-ph.CO].

[21] F. Beutler, C. Blake, M. Colless, D. H. Jones, L. Staveley-Smith, L. Campbell, Q. Parker and W. Saunders et al., Mon. Not. Roy. Astron. Soc. 416 (2011) 3017.

[22] A. J. Ross, L. Samushia, C. Howlett, W. J. Percival, A. Burden and M. Manera, Mon. Not. Roy. Astron. Soc. 449 (2015) 1, 835.

[23] L. Anderson et al. [BOSS Collaboration], Mon. Not. Roy. Astron. Soc. 441 (2014) 1, 24.

[24] A. Cerioni, F. Finelli, A. Tronconi and G. Venturi, Phys. Lett. B 681 (2009) 383.

[25] J. D. Barrow and K. i. Maeda, Nucl. Phys. B 341 (1990) 294.

[26] A. Lewis, "CAMB notes", http://cosmologist.info/notes/CAMB.pdf.

[27] J. Lesgourgues, arXiv:1104.2932 [astro-ph.IM].

[28] D. Blas, J. Lesgourgues and T. Tram, JCAP 1107 (2011) 034.

[29] B. Audren, J. Lesgourgues, K. Benabed and S. Prunet, JCAP 1302 (2013) 001.

[30] Y. C. Li, F. Q. Wu and X. Chen, Phys. Rev. D 88 (2013) 084053.

[31] W. W. Zhu et al., Astrophys. J. 809, no. 1, 41 (2015).

[32] A. G. Riess, L. Macri, S. Casertano, H. Lampeitl, H. C. Ferguson, A. V. Filippenko, S. W. Jha and W. Li et al., Astrophys. J. 730 (2011) 119 [Astrophys. J. 732 (2011) 129].

[33] G. Efstathiou, Mon. Not. Roy. Astron. Soc. 440 (2014) 2, 1138.

[34] A. Avilez and C. Skordis, Phys. Rev. Lett. 113 (2014) 011101.

[35] J. X. Li, F. Q. Wu, Y. C. Li, Y. Gong and X. L. Chen, Res. Astron. Astrophys. 15 (2015) 12, 2151

[36] C. Bambi, M. Giannotti and F. L. Villante, Phys. Rev. D 71 (2005) 123524.

[37] C. J. Copi, A. N. Davis and L. M. Krauss, Phys. Rev. Lett. 92 (2004) 171301.

[38] O. Pisanti, A. Cirillo, S. Esposito, F. Iocco, G. Mangano, G. Miele and P. D. Serpico, Comput. Phys. Commun. 178 (2008) 956.

[39] J. Hamann, J. Lesgourgues and G. Mangano, JCAP 0803 (2008) 004.

[40] B. Bertotti, L. Iess and P. Tortora, Nature 425 (2003) 374.

[41] R. Laureijs et al. [EUCLID Collaboration], arXiv:1110.3193 [astro-ph.CO].

[42] L. Amendola et al. [Euclid Theory Working Group Collaboration], Living Rev. Rel. 16 (2013) 6 [arXiv:1206.1225 [astro-ph.CO]]. 